\title{
Familial Autoimmune Thyroid Disease as a Risk Factor for Regression in Children with Autism Spectrum Disorder: A CPEA Study
}

\author{
Cynthia A. Molloy, ${ }^{1,14}$ Ardythe L. Morrow, ${ }^{1}$ Jareen Meinzen-Derr, ${ }^{1}$ Geraldine Dawson, ${ }^{2}$ \\ Raphael Bernier, ${ }^{2}$ Michelle Dunn, ${ }^{3}$ Susan L. Hyman, ${ }^{4}$ William M. McMahon, ${ }^{5}$ \\ Julie Goudie-Nice, ${ }^{5}$ Susan Hepburn, ${ }^{6}$ Nancy Minshew, ${ }^{7}$ Sally Rogers, ${ }^{8}$ Marian Sigman, ${ }^{9}$ \\ M. Anne Spence, ${ }^{10}$ Helen Tager-Flusberg, ${ }^{11}$ Fred R. Volkmar, ${ }^{12}$ and Catherine Lord ${ }^{13}$
}

\begin{abstract}
A multicenter study of 308 children with Autism Spectrum Disorder (ASD) was conducted through the Collaborative Programs of Excellence in Autism (CPEA), sponsored by the National Institute of Child Health and Human Development, to compare the family history of autoimmune disorders in children with ASD with and without a history of regression. A history of regression was determined from the results of the Autism Diagnostic Interview-Revised (ADIR). Family history of autoimmune disorders was obtained by telephone interview. Regression was significantly associated with a family history of autoimmune disorders (adjusted $\mathrm{OR}=1.89$; 95\% CI: $1.17,3.10)$. The only specific autoimmune disorder found to be associated with regression was autoimmune thyroid disease (adjusted $\mathrm{OR}=2.09 ; 95 \% \mathrm{CI}: 1.28,3.41$ ).
\end{abstract}

KEY WORDS: Autism; ASD; family history; autoimmune; regression.

Autism Spectrum Disorder (ASD) is a neurodevelopmental disorder occurring at a rate of approximately $4 / 1000$, and affecting four times more

\footnotetext{
${ }^{1}$ Center for Epidemiology and Biostatistics, Cincinnati Children's Hospital Medical Center, University of Cincinnati College of Medicine, Cincinnati, Ohio, USA.

${ }^{2}$ University of Washington, Seattle, Washington, USA.

${ }^{3}$ Albert Einstein College of Medicine of Yeshiva University, Bronx, NY, USA.

${ }^{4}$ School of Medicine and Dentistry, University of Rochester, Rochester, NY, USA.

${ }^{5}$ School of Medicine, University of Utah, Salt Lake City, Utah, USA.

${ }^{6}$ Health Sciences Center, University of Colorado, Denver, Colorado, USA.

${ }^{7}$ School of Medicine, University of Pittsburgh, Pittsburgh, PA, USA.

${ }^{8}$ University of California - Davis, Davis, CA, USA.

${ }^{9}$ University of California - Los Angeles, Los Angeles, CA, USA.

${ }^{10}$ University of California - Irvine, Irvine, CA, USA.

${ }^{11}$ Boston University, Boston, MA, USA.

12 Yale University, New Haven, CT, USA.
}

boys than girls (Fombonne, 1999; Yeargin-Allsopp et al., 2003). The diagnosis of autism (autistic disorder) requires that specific clinical criteria be met, as outlined in the Diagnostic and Statistical Manual of the American Psychiatric Association, Fourth Edition within the overarching category of

Abbreviation: CPEA, Collaborative Programs of Excellence in Autism; NICHD, National Institute of Child Health and Human Development; NIDCD, National Institute on Deafness and Other Communication Disorders; ADI-R, Autism Diagnostic InterviewRevised; ADOS, Autism Diagnostic Observation Schedule; ASD, Autism Spectrum Disorder; PDD-NOS, Pervasive Developmental Disorder-Not Otherwise Specified; AITD, Autoimmune Thyroid Disease

13 University of Michigan, Ann Arbor, MI, USA.

${ }^{14}$ Correspondence should be addressed to: Cynthia A. Molloy, Center for Epidemiology and Biostatistics, Cincinnati Children's Hospital Medical Center, University of Cincinnati College of Medicine, 3333 Burnet Avenue, MLC 4051, Cincinnati, OH, 45229-3039, USA; Tel: (513) 636-7301; Fax: (513) 636-7509; e-mail: cynthia.molloy@cchmc.org 
Pervasive Developmental Disorders (PDD) (American Psychiatric Association, 2000). These criteria include deficits in communication and reciprocal social interaction, and the presence of restricted interests or stereotypic behaviors. The term Autism Spectrum Disorder (ASD) is a broader category, inclusive of individuals with autism and those having the same core deficits to a lesser degree of severity (Tanguay, 2000). This latter group includes children diagnosed with Pervasive Developmental Disorder - Not Otherwise Specified (PDD-NOS) and Asperger's Syndrome (American Psychiatric Association, 2000).

While the etiology of ASD is unknown, there is some evidence for an association with immune dysfunction (van Gent, Heijnen, \& Treffers, 1997). There are reports that children with ASD have abnormalities in the peripheral blood cells and cytokines that mediate both innate and adaptive responses (Connolly et al., 1999; Croonenberghs, Bosmans, Deboutte, Kenis, \& Maes, 2002a; Gupta, Aggarwal, Rashanravan, \& Lee, 1998; Jyonouchi, Sun, \& Itokazu, 2002; Singh, 1996; Warren, Foster, \& Margaretten, 1987). Within the adaptive immune response, elevated levels have been reported for both $\mathrm{T}_{\mathrm{H}} 1$ cytokines, commonly seen in autoimmune disorders (Singh, 1996), and $\mathrm{T}_{\mathrm{H}} 2$ cytokines, as seen in atopic diseases (Gupta, Aggarwal, \& Heads, 1998). Elevated serum immunoglobulins (Croonenberghs et al., 2002b) and autoantibodies to neuronal elements (Singh, Warren, Averett, \& Ghaziuddin, 1997) have also been reported in children with autism. In addition, Comi et al. reported that $66 \%$ of children with autism had at least one relative with an autoimmune disorder, compared to $50 \%$ of normally developing controls (Comi, Zimmerman, Frye, Law, \& Peeden, 1999). Sweeten and colleagues also reported a significantly higher mean number of autoimmune disorders in families of children with PDDs $(1.87 \pm 1.6)$ compared to families of healthy controls $(0.93 \pm 1.1)$ or children with known autoimmune disorders $(1.44 \pm 1.5)$ (Sweeten, Bowyer, Posey, Halberstadt, \& McDougle, 2003). Croen and colleagues reported an increased occurrence of asthma and allergy in the mothers of children with ASD, as well as two autoimmune disorders, psoriasis and Type I diabetes (Croen, Yoshida, Odouli, \& Grether 2004).

The evidence for an association between the immune system and ASD is increasing, but no consistent pattern has yet been identified. While there is evidence to support ASD as an autoimmune phenomenon which is commonly associated with a cell-mediated, $\mathrm{T}_{\mathrm{H}} 1$ response (Comi, Zimmerman, Frye, Law, \& Peeden, 1999; Singh, Warren, Averett, Ghaziuddin, 1997; Sweeten, Bowyer, Posey, Halberstadt, \& McDougle, 2003) there is also evidence that the disorder is associated with an allergic $\left(\mathrm{T}_{\mathrm{H}} 2\right)$ immune response (Lucarelli, et al., 1995; Gupta, Aggarwal, Rashanravan, \& Lee, 1998), or that ASD is associated with general dysregulation of the immune system (Gupta, Aggarwal \& Heads, 1996; Jyonouchi, Sun, \& Le, 2001). These conflicting results may be due in part to the underlying heterogeneity within ASD, a diagnosis based on observable clinical and historical findings. Within this clinically defined disorder there are likely to be distinctive subgroups that can provide more homogeneous samples for etiologic studies. Categorizations for some of these subgroups have been based on language skills (Bradford et al., 2001), neurocognitive abilities (Tager-Flusberg \& Joseph, 2003) or the presence of dysmorphic features (Miles \& Hillman, 2000). Another clinical feature with potential immunologic associations is a history of regression (Richler, et al., 2004).

Regression is a phenomenon in which a child loses already established skills in communication or social interaction (Goldberg, et al., 2003). It is reported to occur in $20-30 \%$ of ASD cases, most often between 18 and 24 months of age (Davidovitch, Glick, Holtzman, Tirosh, \& Safir, 2000; Fombonne, 1999; Rapin \& Katzman, 1998; Shinnar et al., 2001), and is rarely seen in other developmental disorders. However, regression itself is, as yet, not clearly defined. Regression may involve loss of language, loss of other socio-communicative behaviors, or both (Goldberg et al., 2003). The majority of children with ASD who regress do not have completely normal development prior to the regression (Lord, Shulman, \& DiLavore, 2004; Siperstein \& Volkmar, 2004) and classification into regressionnonregression groups is dependent upon the definition of regression.

Findings have been mixed on whether or not measurable differences exist between children who experience regression and those who do not. One retrospective review of home videotapes indicated that, at 12-18 months, children who experienced regression had better social behavior, gaze, expressive language and level of play when compared to children with ASD who did not experience regression (Goldberg et al., 2003). In the Collaborative Programs of Excellence in Autism (CPEA) sample used for the current study, Luyster et al. found that children with 
regression were more likely than children with no regression to have early prosocial skills in the typical range (Luyster et al., 2005).

Several investigators have reported a difference in cognitive ability between regression and nonregression groups at school age, finding lower mean IQ scores in children with a history of regression (Burack \& Volkmar, 1992; Kobayashi \& Murata, 1998; Kurita, Kita, \& Miyake, 1992). In their report citing no evidence for a new variant of MMR-induced autism, Fombonne and Chakrabarti found no differences between regression and nonregression groups on symptom severity as measured by ADI scores, but they did report a difference in cognitive functioning that approached significance, with $46.1 \%$ of children in the regression group having an IQ $<70$ compared to $21.5 \%$ of the nonregression group $(p=0.08$, Fisher's exact test) (Fombonne \& Chakrabarti, 2001). Brown and Prelock (1995) also reported that individuals with autism and regression had poorer social skills later in life compared to those with autism and no regression.

Taken together, these findings suggest that a significant minority of children with ASD who experience regression may represent a reasonably homogeneous subgroup for further etiologic research. The purpose of this study was to determine if children with ASD and regression differed from those with ASD and no regression in the frequency of familial autoimmune disorders. Such group differences would support the continued investigation of regression as a marker for a subgroup within ASD with potential immunologic associations.

\section{METHODS}

This study was conducted as part of a larger project within the Collaborative Programs of Excellence in Autism (CPEA). The CPEA is a nationwide collaboration established by The National Institute of Child Health and Human Development (NICHD) and the National Institute on Deafness and Other Communication Disorders (NIDCD). Ten sites participate in the CPEA and were collaborators in the present study: the Albert Einstein College of Medicine, Boston University, University of California - Irvine Medical Center, University of California - Los Angeles, University of California Davis/M.I.N.D Institute and University of Colorado, University of Rochester Medical Center, University of Utah - Utah Autism Project,
University of Washington, University of Pittsburgh - Western Psychiatric Institute and Clinic, and the Yale Child Study Center, which included families recruited through the University of Chicago, the University of North Carolina and the University of Michigan.

Investigators at each of the CPEA sites enroll children with ASD into research studies on an ongoing basis. Data that are part of the standard CPEA evaluation include the Autism Diagnostic Interview - Revised (ADI-R) (Lord, Rutter, \& Le Couteur, 1994), the Autism Diagnostic Observation Schedule (ADOS) (Lord et al., 2000), standard measures of verbal and nonverbal IQ, including the Differential Abilities Scale and the Mullen Scales of Early Learning, and demographic information.

Subjects for this study were identified through the CPEA site databases, which included a total of 1592 children with an ASD diagnosis. Children were eligible for the study if they had a diagnosis of ASD, available records of assessments performed at or near the time of diagnosis, complete ADI-R, ADOS, verbal and nonverbal IQ scores within the last five years. Caretakers had to be available for a follow-up telephone interview, and the children had to be between the ages of 4 and 15 at the time of this interview. Specific selection criteria and methods for standardization across sites have been described previously (Luyster et al., 2005). Since regression is reported to occur in approximately $30 \%$ of children with ASD, the design of the study required over sampling children who experienced regression to result in roughly equal groups of children with a history of regression and children with no history of regression. Attempts were made at each CPEA site to recruit all eligible children identified as having a history of word loss on their initial ADI-R. When enrollment of children with word loss exceeded enrollment of children with no word loss, the coordinating site (Michigan) maintained balance between group sizes by intermittent compensatory over sampling of children with no word loss at that site.

The caretakers of potential subjects were invited to participate in a standardized telephone interview designed for this study and administered by trained interviewers blind to the child's regression history. The interview collected additional information about the child's social and communication skill development and the medical history of the child and family. If there was a discrepancy between the initial ADI-R coding and the telephone interview in reported loss history, the original ADI-R protocol notes were 
reviewed and compared with the interview results. If the loss had been miscoded in the ADI-R (protocol notes confirmed loss but item coded as no loss) the child's classification for regression status was changed. If no supporting evidence in the original ADI-R was found to confirm the telephone interview, the classification was maintained.

A positive history of regression was defined as a loss of any previously acquired social or communication skills, based on all items from the initial ADI$\mathrm{R}$ pertaining to loss of language or other social skills. A subset of the regression group was identified as experiencing word loss. This was defined as having at least three meaningful words used spontaneously on a daily basis for at least a month, followed by a period of at least a month in which no spontaneous language at all was produced.

In addition to other medical history items reported elsewhere (Richler, Luyster et al., 2005), respondents were asked who, if anyone, in the child's biological family had a diagnosis of specific autoimmune disorders, including Type I Diabetes, Autoimmune Thyroid Disease (AITD), Connective Tissue Autoimmune Disease, Juvenile Rheumatoid Arthritis, Rheumatoid Arthritis, Multiple Sclerosis, Vitiligo or Inflammatory Bowel Disease (Crohn's Disease or Ulcerative Colitis) or any other autoimmune disorder. Only first and second degree relatives were included in the final analysis since caregivers were more likely to have accurate knowledge regarding the medical histories of immediate family members.

\section{ANALYSIS}

Two groups were defined by a history of regression established in accordance with the CPEA protocol. The Case group was defined as children with ASD and a history of regression. The Control group was defined as children with ASD and no history of regression. Within the case group we examined separately those children with and without word loss. Proportions in the two groups were compared using the $\chi^{2}$ statistic. Mean scores on standardized tests were compared using $t$-tests and Analysis of Variance (ANOVA). Unadjusted odds ratios were calculated for familial history of autoimmune disorder in general and for specific disorders in cases compared to controls. Potential covariates and confounders were identified, including age at ADI-R, race, gender, diagnosis (autism vs. other PDD), verbal IQ and maternal education. Maternal education was reported as (1) college graduate, (2) some college or associate degree, (3) high school graduate or (4) did not finish high school, GED. After demonstrating that groups 1 and 2 and groups 3 and 4 were similar, a dichotomous variable was created for maternal education: some college or no college.

Logistic Regression models were built to evaluate the contribution of each of the variables to the outcome measure of regression status. Variables that were associated with the regression outcome at the $p \leq 0.1$ level, maternal education and diagnosis, were kept in the final models to calculate the adjusted odds ratios for familial autoimmunity. The potential effect modification (interaction) of familial autoimmunity by maternal education and familial autoimmunity by diagnosis were tested in the model and found not to be significant in relation to the regression outcome $(p>0.2)$. Because $70 \%$ of the sample was recruited from the Michigan/Chicago/NC site, with a higher proportion (76\%) of no word loss subjects (Table I), CPEA site of enrollment was also included in the final model. To evaluate the impact of the classification status of the 35 children who experienced regression without word loss, analyses were repeated with those children removed from the dataset. All statistical analyses were performed using Statistical Analysis System (SAS) statistical package, version 8e (SAS Institute, Inc. 1999).

\section{RESULTS}

Complete data on regression status and familial autoimmune disorders were available on 308 children with ASD from 9 CPEA sites. Demographic information for this sample is shown in Table I. The majority of the subjects (70\%) were recruited through studies conducted at the Chicago/NC/Michigan sites as part of the Yale CPEA. The response rate was evaluated at the Michigan site, where $55 \%$ of the families who were contacted chose to participate in the interview portion of the study (Luyster et al., 2005). There were no significant differences between responders and nonresponders with regard to age, race, gender, diagnosis, maternal education or history of regression from original ADI-R. As reported by Luyster et al. 19\% of children had discrepant information between the original ADI-R and the telephone interview. Forty-three $(12.3 \%)$ children were reported to have word loss in the interview only. On review of original ADI-R data, 6 of these were reclassified. Twenty-three children $(6.6 \%)$ were 
Familial Autoimmune Thyroid Disease

Table I. Characteristics of Sample by History of Regression or NRegression

\begin{tabular}{|c|c|c|c|c|}
\hline & $\begin{array}{c}\text { Regression } n=155 \\
n(\%)\end{array}$ & $\begin{array}{c}\text { No regression } n=153 \\
n(\%)\end{array}$ & Total & $p$ value \\
\hline Male & $134(86)$ & $125(82)$ & $259(84)$ & $0.25^{b}$ \\
\hline Caucasian & $130(84)$ & $124(81)$ & $254(82)$ & $0.51^{b}$ \\
\hline \multicolumn{5}{|l|}{ Diagnosis } \\
\hline Autistic Disorder & $122(79)$ & $104(68)$ & $226(73)$ & $0.03^{b}$ \\
\hline Other $P D D^{a}$ & $33(21)$ & $49(32)$ & $82(27)$ & \\
\hline Maternal education includes some college & $142(92)$ & $126(82)$ & $268(87)$ & $0.02^{b}$ \\
\hline Chicago/NC/Michigan CPEA site & $100(65)$ & $116(76)$ & $216(70)$ & $0.03^{b}$ \\
\hline Verbal IQ Mean \pm SD & $(n=140) 56.8 \pm 33.2$ & $(n=144) 62.5 \pm 31.2$ & $(n=284) 59.7 \pm 32.3$ & $0.14^{c}$ \\
\hline Nonverbal IQ Mean \pm SD & $(n=144) 72.6 \pm 29.5$ & $(n=146) 74.3 \pm 29.3$ & $(n=290) 73.5 \pm 29.3$ & $0.87^{c}$ \\
\hline Age at first ADI (mos) Mean \pm SD & $72.3 \pm 31.0$ & $72.8 \pm 31.7$ & $72.6 \pm 31.3$ & $0.1^{c}$ \\
\hline
\end{tabular}

${ }^{a}$ Other PDD includes ASD, PDD-NOS and Asperger's Syndrome.

${ }^{b} \chi^{2}$ statistic.

${ }^{c} t$-test.

reported to have no word loss at the time of interview. After review, 5 of these were reclassified. Thus, following review of all available data, only $3 \%$ of the children had a change in regression classification from their initial ADI-R.

By design, one half of the children in this study $(n=155)$ experienced regression. For 120 (39\%) children, this regression included word loss. There were no significant differences between the 155 children with regression and the 153 children with no regression in regard to gender, race, IQ scores, diagnosis or age at first ADI. A history of regression was reported significantly more often by mothers with at least some college education $(p=0.02)$, and the larger proportion of children experiencing regression who were diagnosed with autistic disorder approached significance $(p=0.06)$ (Table I).

A total of 175 of the 308 families (57\%) in the sample reported at least one first or second degree relative with an autoimmune disorder. Children with ASD and a history of regression were significantly more likely than children with no history of regression to have a first or second degree relative with an autoimmune disorder (Table II). Among the probands themselves, one child had a history of JRA with no history of regression and one child had AITD with a history of regression. Six children had a diagnosis of inflammatory bowel disease. Of these, 5

Table II. Family History of Autoimmune Disorders in a First or Second Degree Relative as a Risk Factor for Regression in Children with ASD

\begin{tabular}{|c|c|c|c|c|}
\hline & $\begin{array}{c}\text { Regression (any loss) } \\
n=155 \\
n(\%)\end{array}$ & $\begin{array}{c}\text { No Regression } \\
n=144 \\
n(\%)\end{array}$ & $\begin{array}{c}\text { Adjusted }^{a} \text { Odds Ratio } \\
\text { (95\% Confidence Interval) }\end{array}$ & $p$ value \\
\hline $\begin{array}{l}\text { Family History of } 1 \text { or more relatives with } \\
\text { an Autoimmune Disorder }{ }^{b}\end{array}$ & $108(70)$ & $81(55)$ & $1.89(1.17,3.10)$ & 0.009 \\
\hline Type I Diabetes ${ }^{c}$ & $25(16)$ & $24(17)$ & $1.23(0.73,2.10)$ & 0.44 \\
\hline Autoimmune Thyroid Disease $^{c}$ & $64(41)$ & $38(27)$ & $2.09(1.28,3.41)$ & 0.003 \\
\hline Connective Tissue Disease $^{c}$ & $14(9)$ & $10(7)$ & $1.17(0.55,2.48)$ & 0.68 \\
\hline Juvenile Rheumatoid Arthritis ${ }^{c}$ & $4(3)$ & $6(3)$ & $0.60(0.21,1.72)$ & 0.34 \\
\hline Rheumatoid Arthritis ${ }^{c}$ & $39(25)$ & $28(20)$ & $1.14(0.68,1.90)$ & 0.62 \\
\hline Multiple Sclerosis $^{c}$ & $3(3)$ & $2(1)$ & $1.76(0.70,4.42)$ & 0.23 \\
\hline Vitiligo $^{c}$ & $4(3)$ & $6(3)$ & $2.61(0.66,10.28)$ & 0.17 \\
\hline Inflammatory Bowel Disease $^{c}$ & $19(12)$ & $15(10)$ & $1.25(0.60,2.63)$ & 0.55 \\
\hline $\begin{array}{l}\text { Family History of } 2 \text { or more relatives with } \\
\text { an Autoimmune Disorder }{ }^{b}\end{array}$ & $55(35)$ & $47(32)$ & $1.26(0.77,2.06)$ & 0.36 \\
\hline
\end{tabular}

\footnotetext{
${ }^{a}$ Adjusted for diagnosis, maternal education and CPEA site.

${ }^{b}$ Missing data on family history of any autoimmune disease $n=9$.

${ }^{c}$ Missing data on specific autoimmune diseases range $n=10-15$.
} 
had a history of regression. The association between regression and bowel symptoms in this study is reported elsewhere (Richler et al., 2004).

Of the eight specific autoimmune disorders included in the telephone survey, Type I Diabetes, AITD, Connective Tissue Disease, JRA, Rheumatoid Arthritis, Multiple Sclerosis, Vitiligo and Inflammatory Bowel Disease, only familial AITD was found to be a significant risk factor for regression in children with ASD with an odds ratio of $2.09(95 \% \mathrm{CI}=1.28$, 3.41 ) adjusted for maternal education, diagnosis and site (Table II). The odds ratio increased to 2.40 (95\% $\mathrm{CI}=1.38,4.20)$ if the AITD was reported to occur in a maternal first or second degree relative, ie. mother, maternal aunt or uncle, maternal half sibling or maternal grandparent. (Table III). The relationship between regression and AITD in a maternal relative did not vary by the gender of the proband.

With increased specificity, comparing children with word loss $(n=120)$ to children with no regression $(n=153)$, the strength of association for any autoimmune disorder increased to an adjusted odds ratio of 2.16 (95\% CI: 1.24, 3.56). For AITD the adjusted odds ratio increased to 2.36 (95\% CI: 1.40, 3.99) for any relative and 2.96 (95\% CI: 1.65, 5.30; $p=0.0003$ ) for a maternal relative.

\section{DISCUSSION}

In this large sample of well characterized cases of ASD where the definition of regression was standardized across established autism research centers, familial autoimmunity, specifically AITD was a significant risk factor for regression as defined here.
Previous case-control studies have found familial autoimmunity to be a risk factor for autism (Comi et al., 1999; Sweeten et al., 2003). In our study we found that, in children diagnosed with ASD, a history of familial autoimmunity, specifically AITD is a significant risk factor for regression, suggesting that this group of children with ASD and regression may contribute substantially to the associations that have been described.

In this study, an association was found between a specific autoimmune disorder, AITD, and regression in children with ASD. Among all autoimmune disorders, AITD is the most common, with a prevalence estimate of 2-4\% in women and $1 \%$ in men (Vaidya, Kendall-Taylor, \& Pearce, 2002). We did not find associations with any other specific autoimmune disorders, but even with an estimated 3100 total relatives in this study, we may not have had sufficient power to detect differences in the less common autoimmune disorders. The reports of an increased prevalence of AITD and other immune disorders among relatives of patients with rheumatoid arthritis (Walker, Griffiths, \& Griffiths, 1986), Type I Diabetes (Payami, Joe, \& Thomson, 1989), idiopathic inflammatory myopathy (Ginn et al., 1998), multiple sclerosis (Broadley, Deans Sawcer, Clayton, \& Compston, 2000) and JRA (Prahalad, Shear, Thompson, Giannini, \& Glass, 2002) have been interpreted as evidence for a common polygenic risk factor that confers susceptibility to autoimmunity (Ginn et al., 1998).

The increased risk for regression in children with ASD having a relative with AITD may also result from these genetic risk factors common to autoimmune disorders. Alternatively, there may be disease

Table III. Family History of Autoimmune Thyroid Disease as a Risk Factor for Regression in Children with ASD

\begin{tabular}{|c|c|c|c|c|}
\hline & $\begin{array}{c}\text { Regression (any loss) } \\
n=155 \\
n(\%)\end{array}$ & $\begin{array}{c}\text { No Regression } \\
n=141 \\
n(\%)\end{array}$ & $\begin{array}{c}\text { Adjusted }^{a} \text { Odds Ratio } \\
\text { (95\% Confidence Interval) }\end{array}$ & $p$ value \\
\hline $\begin{array}{l}\text { Autoimmune Thyroid Disease in any } \\
\text { first or second degree relative }{ }^{b}\end{array}$ & $64(41)$ & $38(27)$ & $2.09(1.28,3.41)$ & 0.003 \\
\hline $\begin{array}{l}\text { Autoimmune Thyroid Disease in a } \\
\text { maternal first or second degree relative }\end{array}$ & $52(34)$ & $25(18)$ & $2.40(1.38,4.20)$ & 0.002 \\
\hline $\begin{array}{l}\text { Autoimmune Thyroid Disease in a } \\
\text { paternal first or second degree relative }\end{array}$ & $18(12)$ & $17(12)$ & $1.06(0.51,2.18)$ & 0.88 \\
\hline Autoimmune Thyroid Disease in mother & $15(10)$ & $7(5)$ & $2.01(0.78,5.17)$ & 0.15 \\
\hline Autoimmune Thyroid Disease in father & $4(3)$ & $4(3)$ & $0.99(0.24,4.17)$ & 0.99 \\
\hline
\end{tabular}

${ }^{a}$ Adjusted for diagnosis, maternal education and CPEA site.

${ }^{b}$ Missing data $n=12$. 
specific susceptibility allele(s) common to AITD and regression in ASD, or there may be alleles contributing separately to the disorders that are in linkage disequilibrium and track together in families. As these and other hypotheses of immune function in ASD are explored, the results of this study support the segregation of ASD cases by a history of regression as a way to improve sample homogeneity.

In this study, the history of regression and familial autoimmune disorders were obtained through caregiver report, which is a limitation. However, the history of regression was based on the ADI-R that was conducted at the CPEA site at the time of diagnosis, minimizing reliance on recall of past events for classification of cases and controls. The history of familial autoimmunity was obtained through direct interview of the caregiver by telephone. The issue of selective recall of family history that can introduce a potential bias when comparing children with a condition such as ASD to healthy controls is also minimized in this study since both cases and controls had ASD, and were subject to the increased awareness of family history that may result from the diagnosis of a developmental disorder.

Because the nature of regression is not well understood, potential misclassification of the children with regression without word loss is another limitation of the study. When the 35 children with regression and no word loss were eliminated from the analysis, the proportion of children with familial AITD increased from 41 to $46 \%$ in the case group characterized strictly by word loss $(n=120)$. This increased the adjusted odds ratio to $2.36(95 \% \mathrm{CI}$ : $1.40,3.99)$.

Even as regression itself becomes better characterized through ongoing research, attention to the subgroup of children with ASD and a history of regression is warranted in studies of autoimmunity and immune function. With the diagnostic instruments now available, it is possible to confidently characterize more homogeneous samples of children with ASD with and without regression that can be compared for evidence of immune system dysregulation.

\section{ACKNOWLEDGMENTS}

We are grateful to the patients and families at all the CPEA sites who participated in this research. We thank Dr. Mekibib Altaye for his critical review of the statistical analyses. We also thank Dr. Thayne
Sweeten for his thoughtful reading and comments on the manuscript and Deb Anderson for her assistance in data management.

\section{REFERENCES}

American Psychiatric Association. (2000). Diagnostic and Statistical Manual of Mental Disorders (4th ed.). Washington, DC: American Psychiatric Association.

Bradford, Y., Haines, J., Hutcheson, H., Gardiner, M., Braun, T., Sheffield, V., Cassavant, T., Huang, W., Wang, K., Vieland, V., Folstein, S., Santangelo, S., \& Piven, J. (2001). Incorporating language phenotypes strengthens evidence of linkage to autism. American Journal of Medical Genetics, 105(6), 539547.

Broadley, S. A., Deans, J., Sawcer, S. J., Clayton, D., \& Compston, D. A. (2000). Autoimmune disease in first-degree relatives of patients with multiple sclerosis A UK survey. Brain, $123(\mathrm{Pt} 6)$, 1102-1111.

Brown, J., \& Prelock, P. A. (1995). Brief report: The impact of regression on language development in autism. Journal of Autism and Developmental Disorders, 25(3), 305-309.

Burack, J. A., \& Volkmar, F. R. (1992). Development of low- and high-functioning autistic children. Journal of Child Psychology and Psychiatry, 33(3), 607-616.

Comi, A. M., Zimmerman, A. W., Frye, V. H., Law, P. A., \& Peeden, J. N. (1999). Familial clustering of autoimmune disorders and evaluation of medical risk factors in autism. Journal of Child Neurololgy, 14(6), 388-394.

Connolly, A. M., Chez, M. G., Pestronk, A., Arnold, S. T., Mehta, S., \& Deuel, R. K. (1999). Serum autoantibodies to brain in Landau-Kleffner variant, autism, and other neurologic disorders. Journal of Pediatrics, 134(5), 607-613.

Croen, L. A., Yoshida, C. K., Odouli, R., \& Grether, J. K. (2004) Maternal autoimmune and allergic diseases and childhood autism. Presented at the International Meeting for Autism Research, Sacramento, CA. May 7-8.

Croonenberghs, J., Bosmans, E., Deboutte, D., Kenis, G., \& Maes, M. (2002a). Activation of the inflammatory response system in autism. Neuropsychobiology, 45(1), 1-6.

Croonenberghs, J., Wauters, A., Devreese, K., Verkerk, R., Scharpe, S., Bosmans, E., Egyed, B., Deboutte, D., \& Maes, M. (2002b). Increased serum albumin, (globulin, immunoglobulin IgG, and IgG2 and IgG4 in autism. Psychological Medicine, 32, 1457-1463.

Davidovitch, M., Glick, L., Holtzman, G., Tirosh, E., \& Safir, M. P. (2000). Developmental regression in autism: Maternal perception. Journal of Autism and Developmental Disorders, 30(2), $113-119$.

Fombonne, E. (1999). The epidemiology of autism: A review. Psychological Medicine, 29, 769-786.

Fombonne, E., \& Chakrabarti, S. (2001). No evidence for a new variant of measles-mumps-rubella-induced autism. Pediatrics, 108(4), E58.

Ginn, L.R., Lin, J.P., Plotz, P.H., Bale, S.J., Wilder, R.L., Mbauya, A., \& Miller, F.W. (1998). Familial autoimmunity in pedigrees of idiopathic inflammatory myopathy patients suggests common genetic risk factors for many autoimmune diseases. Arthritis and Rheumatism, 41(3), 400-405.

Goldberg, W., Osann, K., Filipek, P., Laulhere, T., Jarvis, K., Modahl, C., Flodman, P., \& Spense, M. (2003). Language and other regression: Assessment and timing. Journal of Autism and Developmental Disorders, 33, 607-616.

Gupta, S., Aggarwal, S., \& Heads, C. (1996). Dysregulated immune system in children with autism: Beneficial effects of intravenous immune globulin on autistic characteristics. Journal of Autism and Developmental Disorders, 26, 439-452. 
Gupta, S., Aggarwal, S., Rashanravan, B., \& Lee, T. (1998). Th1and Th2-like cytokines in CD4 + and CD8 $+\mathrm{T}$ cells in autism. Journal of Neuroimmunology, 85(1), 106-109.

Jyonouchi, H., Sun, S., \& Itokazu, N. (2002). Innate immunity associated with inflammatory responses and cytokine production against common dietary proteins in patients with autism spectrum disorder. Neuropsychobiology, 46(2), 76-84.

Jyonouchi, H., Sun, S., \& Le, H. (2001). Proinflammatory and regulatory cytokine production associated with innate and adaptive immune responses in children with autism spectrum disorders and developmental regression. Journal of Neuroimmunology, 120(1-2), 170-179.

Kobayashi, R., \& Murata, T. (1998). Setback phenomenon in autism and long-term prognosis. Acta Psychiatrica Scandinavica, 98(4), 296-303.

Kurita, H., Kita, M., \& Miyake, Y. (1992). A comparative study of development and symptoms among disintegrative psychosis and infantile autism with and without speech loss. Journal of Autism and Developmental Disorders, 22(2), 175-188.

Lord, C., Risi, S., Lambrecht, L., Jr. Cook, E. H., Leventhal, B. L., DiLavore, P. C., Pickles, A., \& Rutter, M. (2000). The autism diagnostic observation schedule-generic: A standard measure of social and communication deficits associated with the spectrum of autism. Journal of Autism and Developmental Disorders, 30(3), 205-223.

Lord, C., Rutter, M., \& Le Couteur, A. (1994). Autism diagnostic interview-revised: A revised version of a diagnostic interview for caregivers of individuals with possible pervasive developmental disorders. Journal of Autism and Developmental Disorders, 24(5), 659-685.

Lord, C., Shulman, C., \& DiLavore, P. (2004). Regression and word loss in autism spectrum disorder. Journal of Child Psychology and Psychiatry, 45(5), 936-955.

Lucarelli, S., Frediani, T., Zingoni, A. M., Ferruzzi, F., Giardini, O., Quintieri, F., Barbato, M., D’Eufemia, P., \& Cardi, E. (1995). Food allery and infantile autism. Panminerva Medicine, 37(3), 137-141.

Luyster, R., Richler, J., Risi, S., Hsu, W. L., Dawson, G., Bernier, R., Dunn, M., Hepburn, S., Hyman, S. L., McMahon, W. M., Goudie-Nice, J., Minshew, N., Rogers, S., Sigman, M., Spence, M. A., Goldberg, W. A., Tager-Flusberg, H., Volkmar, F. R., \& Lord, C. (2005). Early regression in social communication in autism spectrum disorders: A CPEA study. Developmental Neuropsychology, 27(3), 311-336.

Miles, J. H., \& Hillman, R. E. (2000). Value of a clinical morphology examination in autism. American Journal of Medical Genetics, 91(4), 245-253.

Payami, H., Joe, S., \& Thomson, G. (1989). Autoimmune thyroid disease in type I diabetic families. Genetic Epidemiology, 6(1), 137-141.

Prahalad, S., Shear, E. S., Thompson, S. D., Giannini, E. H., \& Glass, D. N. (2002). Increased prevalence of familial autoimmunity in simplex and multiplex families with juvenile rheumatoid arthritis. Arthritis \& Rheumatism, 46(7), 1851-1856.
Rapin, I., \& Katzman, R. (1998). Neurobiology of autism. Annals of Neurology, 43(1), 7-14.

Richler, J., Luyster, R., Risi, S., Hsu, W., Dawson, G., Bernier, R., Dunn, M., Hyman, S., McMahon, W., Goudie, J., Minshew, N., Rogers, S., Sigman, M., Spence, M., Tager-Flusberg, H., Volkmar, F., \& Lord, C. (2004). Is there a regressive "phenotype" of Autism Spectrum Disorder associated with the Measles-Mumps-Rubella Vaccine: A CPEA Study. Presented at the International Meeting for Autism Research, Sacramento, CA. May 7-8, 2004.

Shinnar, S., Rapin, I., Arnold, S., Tuchman, R. F., Shulman, L., Ballaban-Gil, K., Maw, M., Deuel, R. K., \& Volkmar, F. R. (2001). Language regression in childhood. Pediatric Neurology, 24(3), 183-189.

Singh, V. K. (1996). Plasma increase of interleukin-12 and interferon-gamma Pathological significance in autism. Journal of Neuroimmunology, 66(1-2), 143-145.

Singh, V. K., Warren, R., Averett, R., \& Ghaziuddin, M. (1997). Circulating autoantibodies to neuronal and glial filamentproteins in autism. Pediatric Neurology 17, 88-90.

Siperstein, R., \& Volkmar, F. (2004). Parental reporting of regression in autism. Journal of Autism and Developmental Disorders, 34(6), 731-734.

Sweeten, T. L., Bowyer, S. L., Posey, D. J., Halberstadt, G. M., \& McDougle, C. J. (2003). Increased prevalence of familial autoimmunity in probands with pervasive developmental disorders. Pediatrics, 112(5), e420.

Tager-Flusberg, H., \& Joseph, R. M. (2003). Identifying neurocognitive phenotypes in autism. Philosophical Transactions of the Royal Society of London. Series B. Biological Sciences, 358(1430), 303-314.

Tanguay, P. E. (2000). Pervasive developmental disorders: a 10year review. Journal of the American Academy of Child and Adolescent Psychiatry, 39(9), 1079-1095.

Vaidya, B., Kendall-Taylor, P., \& Pearce, S. H. (2002). The genetics of autoimmune thyroid disease. Journal of Clinical Endocrinology and Metabolism, 87(12), 5385-5397.

van Gent, T., Heijnen, C. J., \& Treffers, P. D. (1997). Autism and the immune system. Journal of Child Psychology and Psychiatry, 38(3), 337-349.

Walker, D. J., Griffiths, M., \& Griffiths, I. D. (1986). Occurrence of autoimmune diseases and autoantibodies in multicase rheumatoid arthritis families. Annals of Rheumatic Diseases, 45(4), 323-326.

Warren, R. P., Foster, A., \& Margaretten, N. C. (1987). Reduced natural killer cell activity in autism. Journal of the American Academy of Child and Adolescent Psychiatry, 26(3), 333-335.

Yeargin-Allsopp, M., Rice, C., Karapurkar, T., Doernberg, N., Boyle, C., \& Murphy, C. (2003). Prevalence of autism in a US metropolitan area. Journal of the American Medical Association, 289(1), 49-55. 